\title{
Effect of Mulligan Mobilization with Movement versus Kinesiotaping in Frozen Shoulder
}

\author{
Krisha A Shah ${ }^{1}$, Leena Zore ${ }^{2}$, Ajay Kumar ${ }^{3}$ \\ ${ }^{1}$ B.P.Th, DPO's NETT College of Physiotherapy, Thane, Maharashtra, India. \\ ${ }^{2}$ M.P.Th, Assistant Professor, DPO's NETT College of Physiotherapy, Thane. \\ ${ }^{3}$ Principal, DPO's NETT College of Physiotherapy, Thane.
}

Corresponding Author: Krisha A Shah

\begin{abstract}
Background: Frozen Shoulder is a painful shoulder condition with insidious onset that was associated with stiffness and loss of motion in the shoulder joint. Mulligan Mobilization with Movement for shoulder joint combines sustained manual application of 'gliding' force to a joint, with the aim of repositioning the positional faults with concurrent physiological motion of the joint. Kinesiotaping improves glenohumeral motions and reduces microtrauma and mechanical irritation of soft tissue structures and reorient shoulder movements through arc of improved glenohumeral motion.

Objective: To compare effect of Mulligan Mobilization with Movement and Kinesiotaping on pain and active shoulder range of motion using VAS and universal goniometer.

Method: 30 subjects were selected as per inclusion and exclusion criteria and were randomly allocated into 2 groups of 15 each. Group A received Mulligan Mobilization with Movement and Group B received Kinesiotaping for shoulder joint. Pre and Post intervention shoulder external rotation, abduction and internal rotation range of motion and VAS scores were analysed.

Result: The statistical analysis showed that there is a significant increase in range of motion and significant reduction in pain scores post intervention in both the groups $(\mathrm{p}<0.0001)$. However, inter group analysis showed that Group $\mathrm{A}$ is much more effective in improving range of motion and reducing pain scores.

Conclusion: The present study concluded that Mulligan Mobilization With Movement is a better intervention on Frozen shoulder as it shows greater increase in shoulder range of motion and significant pain reduction when compared to Kinesiotaping technique.
\end{abstract}

Keywords: Mulligan Mobilization with Movement (MWM), Kinesiotaping, Frozen shoulder, pain, Range of Motion.

\section{INTRODUCTION}

Frozen shoulder also called as Adhesive capsulitis or Periarthritis of shoulder is characterised by pain and loss of motion of the shoulder joint. ${ }^{[1]}$

It is characterised by progressive loss of both active and passive range of motion of the shoulder joint. ${ }^{[2]}$

Frozen shoulder occurs when the capsule of the joint and the surrounding connective tissue becomes shortened, stiff and inflamed. ${ }^{[3]}$
The incidence of adhesive capsulitis is $3-5 \%$ in general population and $20 \%$ in people with diabetes. The most common limitations of range of motion are shoulder abduction, external rotation and internal rotation. ${ }^{[4]}$

It affects the anteriosuperior joint capsule, axillary recess and the coracohumeral ligament.

$70 \%$ of frozen shoulder patients are women, however males with frozen shoulder are at greater risk for longer recovery and greater disabilities. 
The stages of frozen shoulder are: ${ }^{[4],[5]}$

1. The 'freezing' stage -

Patient presents with spontaneous onset of shoulder pain which is more severe at night and with activities, associated with sense of discomfort that radiates down the arm.

Typically lasts for 10 to 36 weeks.

2. The 'frozen' stage -

Pain at rest usually diminishes during this stage leaving the shoulder with restricted motion in all planes.

Activities of daily living become severely restricted.

When performing the activities, a sharp, acute discomfort can occur as the patient reaches the restraint of the tight capsule. It lasts for 4 to 12 months.

3. The 'thawing' stage -

This stage is characterized by gradual recovery of range of motion.

This phase lasts for 5 to 26 months.

$>$ Conservative treatment of frozen shoulder consists of wide range of procedures such as exercise therapy, local anaesthetic, acupuncture, ice/heat therapy, Interferential therapy, kinesiotaping, joint mobilization procedures, etc.

\section{MULLIGAN MOBILIZATION WITH MOVEMENT:}

Mulligan mobilization with movement (MWM) was invented by Brian Mulligan.

Mulligan Mobilization with Movement for peripheral joints combines sustained manual application of 'gliding' force to a joint, with the aim of repositioning the positional faults with concurrent physiological motion of the joint, either performed actively by the subject or passively by the therapist. ${ }^{[5]}$

Mulligan Mobilization with Movement reduces pain due to neurophysiologic effects on the stimulation of peripheral mechanoreceptors and the inhibition of nociceptors. The activation of apical spinal neurons as a result of peripheral mechanoreceptor by the joint mobilization produces presynaptic inhibition of nociceptive afferent activity.

\section{KINESIOTAPING TECHNIQUE:}

Kinesiotaping was developed by Japanaese Chiropractor Dr.Kenzo Kase in the 1970's with the intention to alleviate pain and improve the healing in soft tissues.

Kinesiotape is a thin, porous elastic cotton tape mimicking human skin with roughly same thickness and inherent elastic properties of epidermis. It is $100 \%$ latex free material with acrylic adhesive which is heat activated. $^{[6]}$

Kinesiotaping technique is based on the simple principle that the body has built in healing mechanisms. The results are increased fluid flow through the injured area, better control over muscle contractions, reduce pain and heal faster.

Kinesiotaping allows movement with corrected scapular alignment during shoulder movement, improves glenohumeral motions and reduces microtrauma and mechanical irritation of soft tissue structures and reorient shoulder movements through arc of improved glenohumeral motion. ${ }^{[7]}$

It can be freely applied to any part of the body and do not limit patients' freedom of movement.

\section{MATERIALS AND METHODOLOGY}

The study was a randomized controlled trial where 30 subjects having frozen shoulder were selected using convenient sampling. Inclusion criteria: subjects willing to participate, age group of 40 to 60 years, subjects with limited range of motion in capsular pattern, VAS more than 4/10. Exclusion criteria: subjects with rotator cuff tear, malignancy, severely osteoporotic, subjects hypersensitive to tape.

\section{PROCEDURE}

A written consent form was taken from the subject in the language best understood by them. Screening of the subject was done as per the inclusion and exclusion criteria. The aim, need of study and procedure was explained to the subjects 
following which the subject were divided into 2 groups, group A and group B of 15 subjects each. Both the groups were given hot pack and active shoulder range of motion exercises with wand for abduction, external and internal rotation. Before application of Mobilization with Movement and Kinesiotaping, a pain score on visual analogue scale, active shoulder range of motion by using full scale universal goniometer was taken. And after application of Mobilization with Movement and Kinesiotaping, pain score on visual analogue scale and active shoulder range of motion by using full scale goniometer was taken.

\section{MULLIGAN'S TECHNIQUE: MWM FOR INTERNAL AND EXTERNAL ROTATION}

- Patient position: supine lying with scapula at the edge of the plinth

- Therapist position: standing lateral to the affected joint.

- Hand placement: patients shoulder and elbow is placed at 90 degrees of flexion. Therapist grasps distal humerus with both hands.

- Belt placement: belt should be close to shoulder joint line as much as possible. Belt is secured around therapist waist and should be parallel to floor and perpendicular to humerus.

Therapist distracts the joint laterally and asks the patient to perform external and internal rotation actively and passive overpressure was applied at end range.

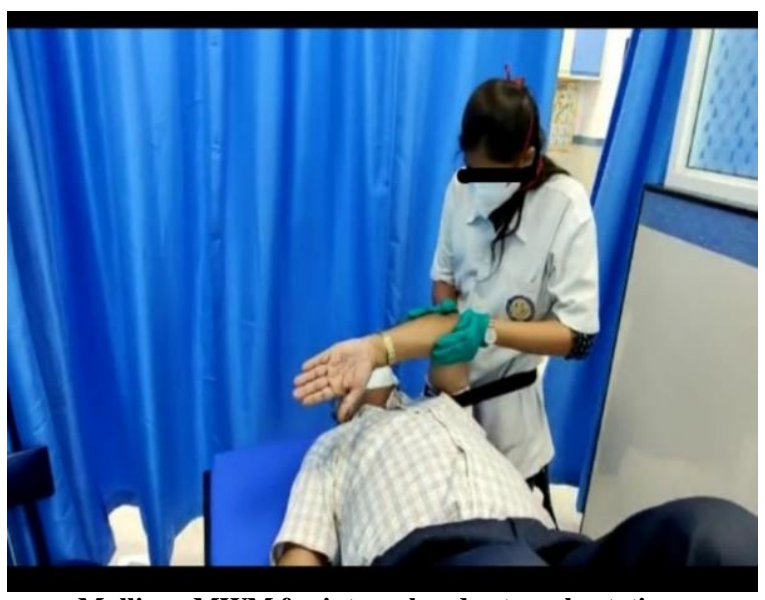

Mulligan MWM for internal and external rotation.

\section{MWM FOR SHOULDER ABDUCTION (POSTEROLATERAL GLIDE):}

- Patient position: sitting on a chair with by the side and head in neutral position.

- Therapist position and procedure: standing posterolateral to the affected side. Therapist places the mulligan belt across the humeral head and to his waist.

- Leaning backward, he applies a posterolateral glide and patient actively performs shoulder abduction, then overpressure was given.

- Maintain the posterolateral glide throughout and return to neutral. Ensure that the movement is pain free.

- Repeat the procedure 3 set of 10 repetitions.

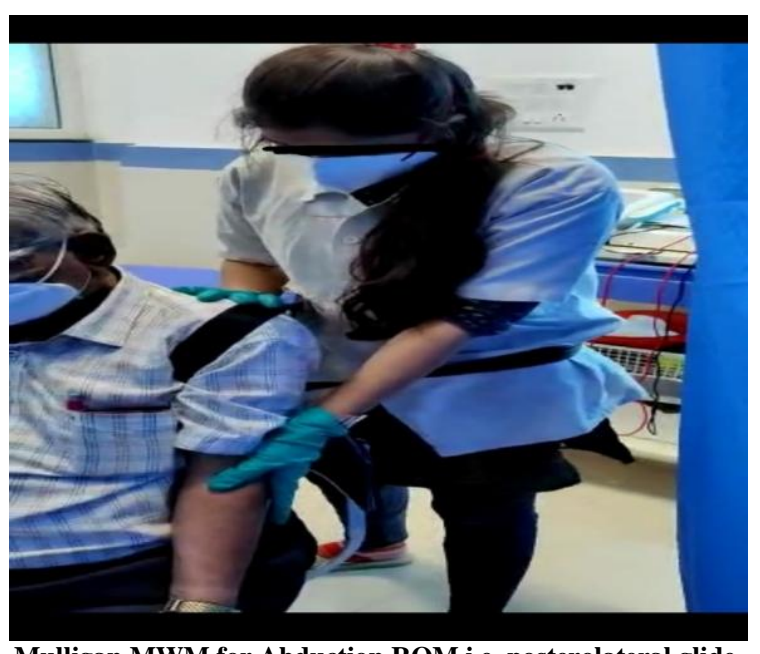

Mulligan MWM for Abduction ROM i.e. posterolateral glide.

\section{KINESIOTAPING TECHNIQUE:}

- Patient position- sitting on a chair with back supported.

- The affected area should be cleaned properly and then dried for proper attachment of kinesiotape.

- Allergy test was performed before the procedure.

\section{For supraspinatus muscle:}

The patient was made to sit on the chair and neck was kept in lateral flexed to opposite side. Affected arm was adducted and internally rotated. A kinesio Y strip of 2 inches was applied from below the greater tuberosity of humerus without tension. A light tension or paper of tension was applied to the $\mathrm{k}$ tape $\mathrm{Y}$ strip. The superior tail was 
applied superior to the spinous process of the scapula between the upper end and middle trapezius muscles and ends at the supraspinous fossa on the superior medial border of scapula. The inferior tail was applied along the spinous process of scapula, the distal 1-2 inches without tension.

\section{For deltoid:}

- Kinesiotape was applied from insertion to origin and Y shaped strip was used.
- Patient in sitting position, the base of kinesiotape Y strip was 2 inches below the deltoid tuberosity of humerus with no tension.

- For anterior deltoid one strip was applied with patients arm in horizontal abduction and external rotation and horizontal extension.

- For posterior deltoid another strip was applied with patients arm in adduction, horizontal flexion and internal rotation.

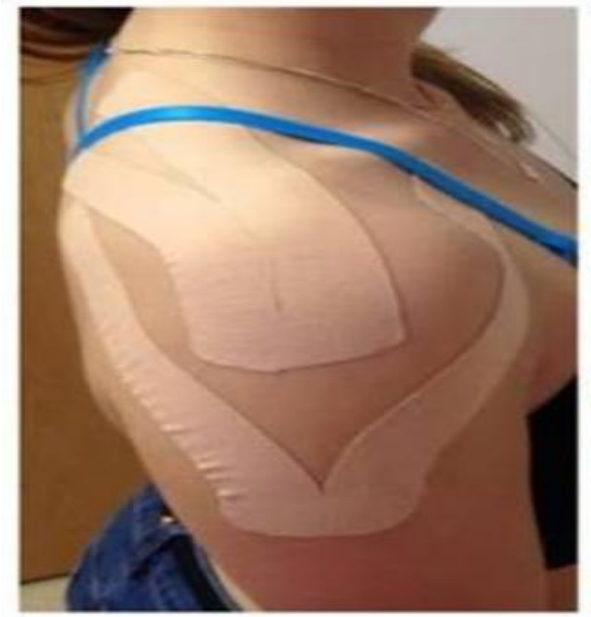

Y strip applied for deltoid i.e. anterior deltoid and posterior deltoid and another Y strip applied for supraspinatus muscle.

\section{Statistical Analysis}

Collected data was entered in Microsoft Excel and Graph Pad Prism 9.2.1 was used for Data Analysis. Normality of the data of range of motion and pain scores for both groups was tested using ShapiroWilk test. Since most of the data was not normally distributed for both range of motion and pain scores for both the groups, statistical analysis was done using nonparametric tests, i.e. for within group comparison was done using Wilcoxon Signed Rank test and inter group comparison was done using the MannWhitney U test for both the groups. Since some groups passed the normality test, statistical analysis was done using parametric tests, i.e. within group was done using paired ' $t$ ' test.

\section{RESULTS}

The result of Mann-Whitney test for inter group comparison shows that Mulligan
Mobilization with Movement is statistically more significant in improving pain and range of motion scores when compared to Kinesiotaping. $(\mathrm{p}<0.0001)$

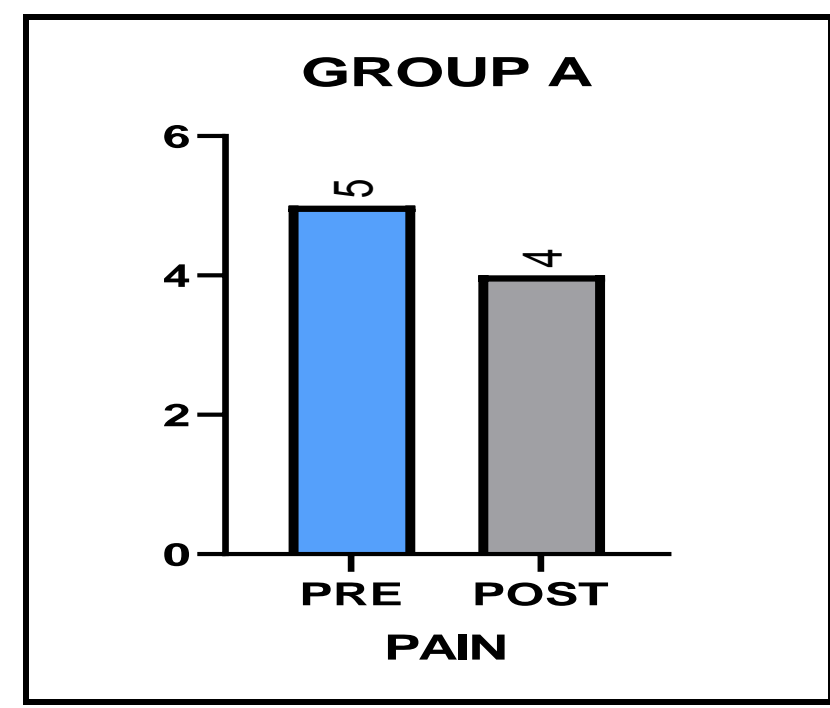

Graph 1 - comparison of pre and post pain scores following the application of Mulligan MWM.

The above graph shows that there was significant reduction in pain scores 
following the application of mulligan $\operatorname{MWM}(\mathrm{P}<0.0001)$

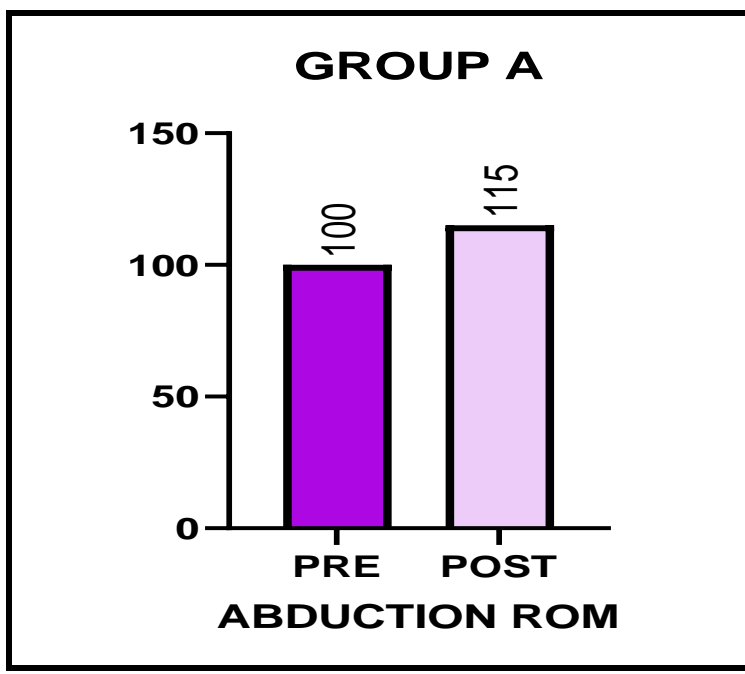

Graph 2 - comparison of pre and post shoulder abduction ROM following the application of Mulligan MWM.

The above graph shows that there was significant increase in shoulder abduction following the application of mulligan MWM ( $\mathrm{p}<0.0001)$.

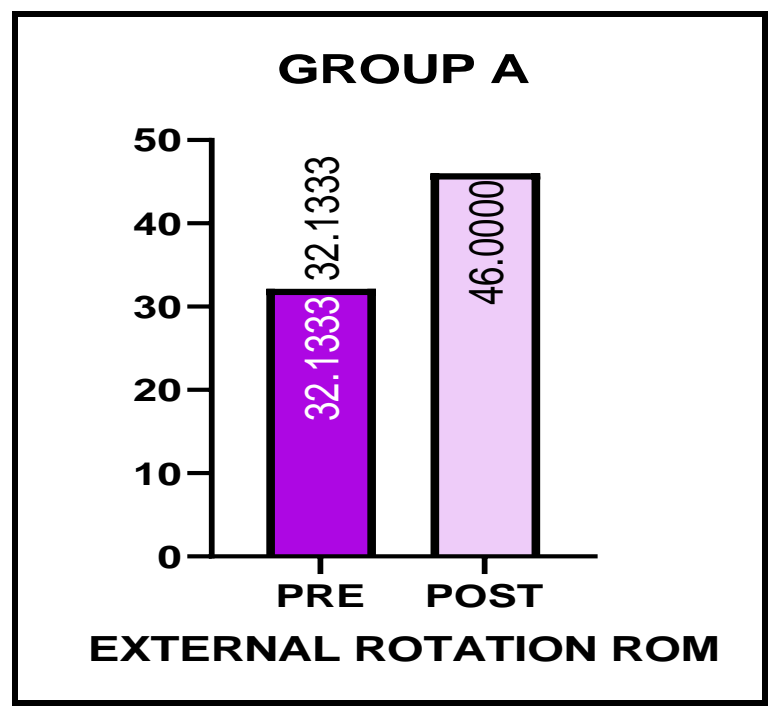

Graph 3- comparison of pre and post shoulder external rotation ROM following the application of Mulligan MWM.

The above graph shows that there was significant increase in shoulder external rotation following the application of mulligan MWM. $(\mathrm{p}<0.0001)$

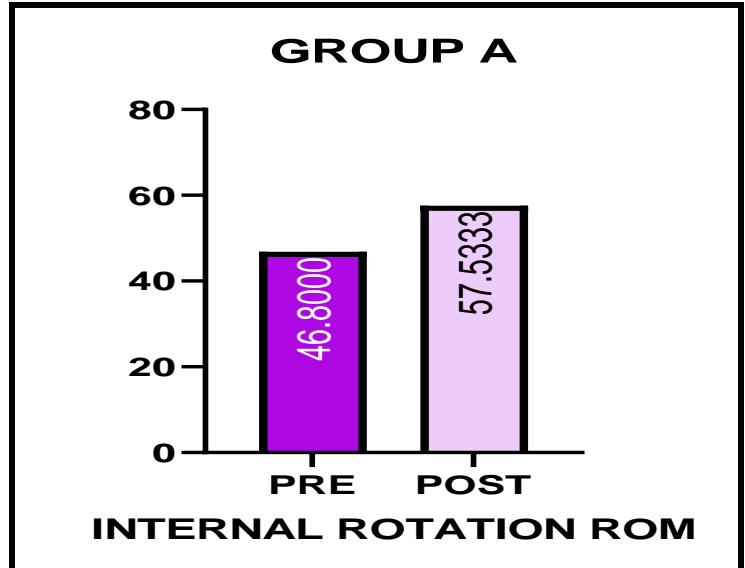

Graph 4- comparison of pre and post shoulder internal rotation following the application of Mulligan MWM.

The above graph shows that there was significant increase in internal rotation following the application of mulligan MWM technique. $(\mathrm{p}<0.0001)$.

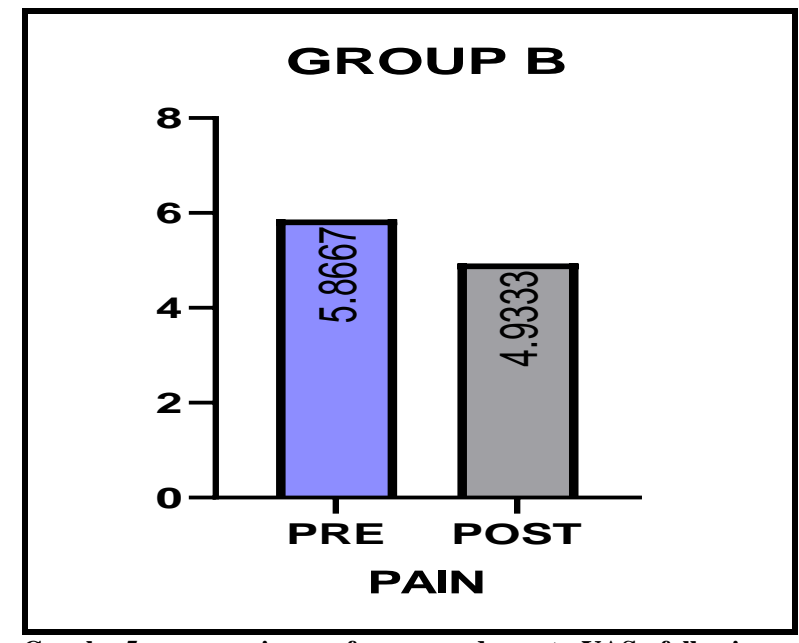

Graph 5- comparison of pre and post VAS following Kinesiotaping technique.

The above graph shows that there was significant reduction in VAS following kinesiotaping technique. $(\mathrm{p}<0.0001)$

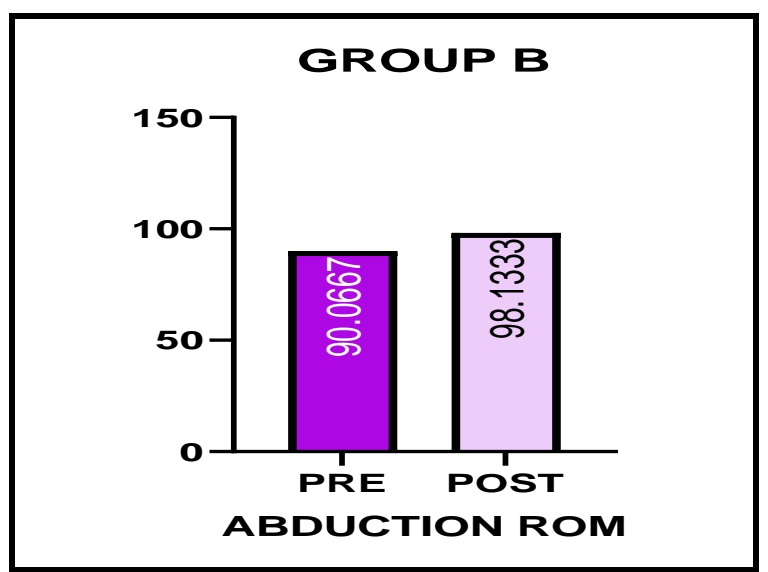

Graph 6- comparison of pre and post shoulder abduction ROM following kinesiotaping technique. 
The above graph shows that there is significant increase in shoulder abduction ROM following the application of kinesiotaping technique. $(\mathrm{p}<0.0001)$

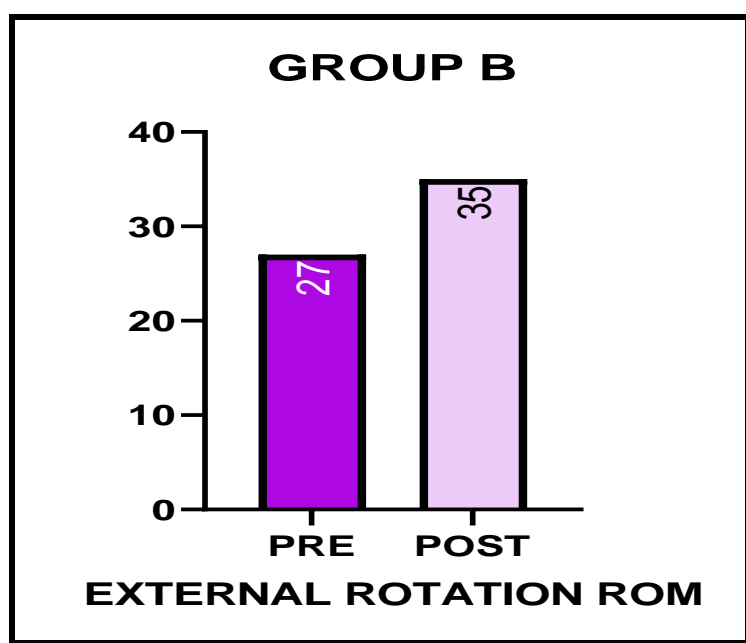

Graph 7- Comparison of pre and post shoulder external rotation following Kinesiotaping technique.

The above graph shows that there was significant increase in shoulder external rotation following the application of kinesiotaping technique. $(\mathrm{p}<0.0001)$

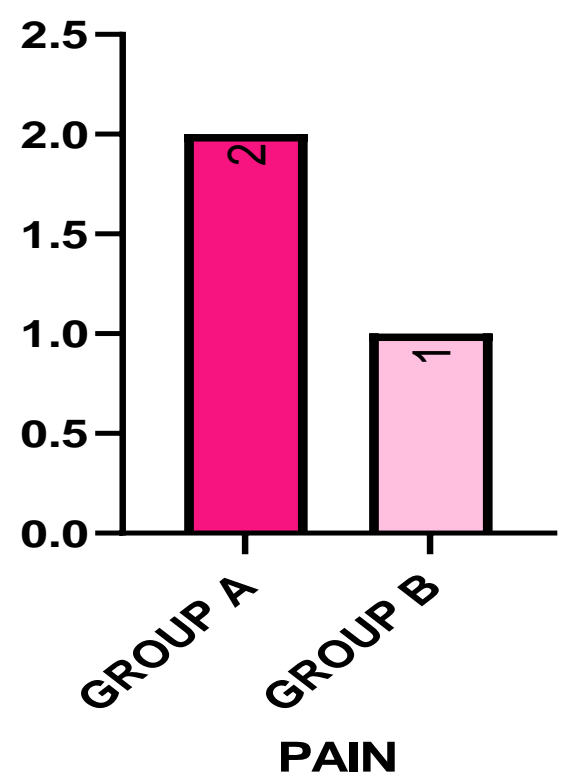

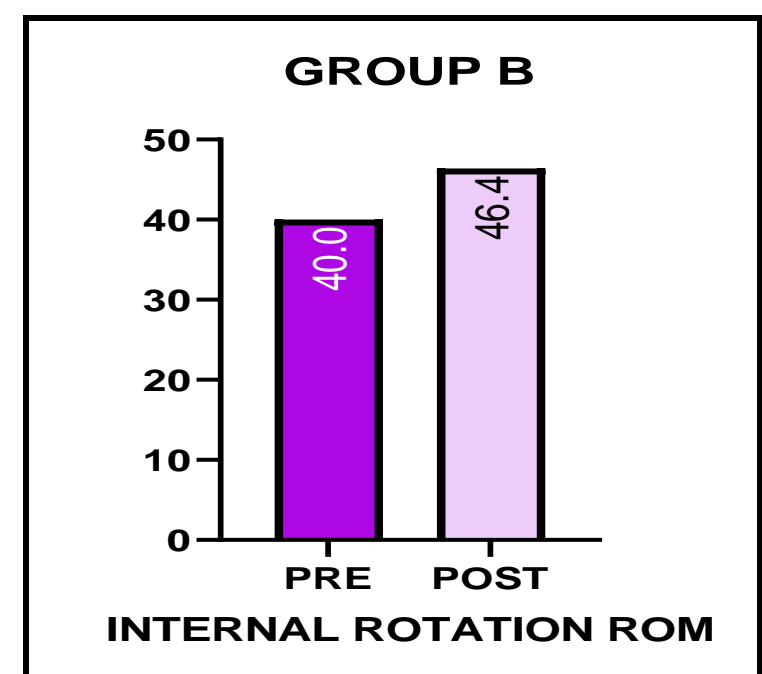

Graph 8- comparison of pre and post shoulder internal rotation following kinesiotaping technique.

The above graph shows that there is significant increase in shoulder internal rotation ROM following the application of kinesiotaping technique. $(\mathrm{p}<0.0001)$

Graph 9- comparison of VAS following Mulligan MWM and kinesiotaping technique.

The above graph shows that there is significant reduction in VAS scores following the application of mulligan MWM technique. $(\mathrm{p}<0.0001)$. 


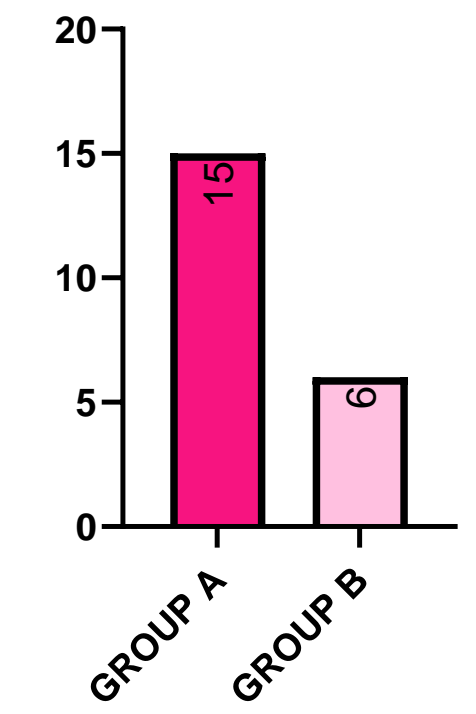

GROUP A (MULLIGAN MWM

GROUP B (KINESIOTAPING)

\section{EXTERNAL ROTATION ROM}

Graph 10- comparison of external rotation ROM following MWM and kinesiotaping.

The above graph shows that there is significant increase in external rotation ROM following the application of mulligan MWM. $(\mathrm{p}<0.0001)$

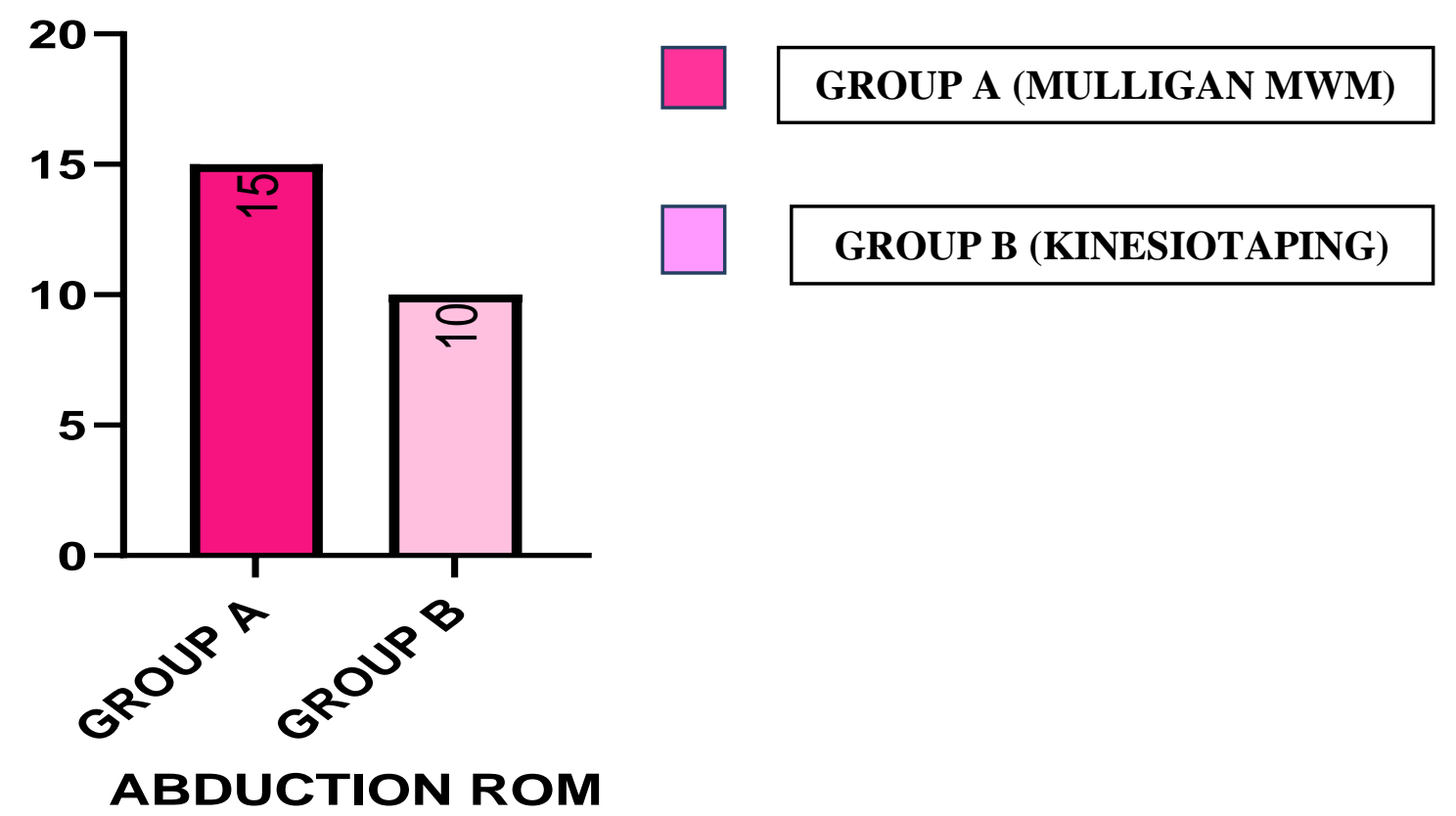

Graph 11- comparison of abduction ROM following MWM and Kinesiotaping technique.

The above graph shows that there is significant increase in abduction ROM following the application of Mulligan MWM. $(\mathrm{p}<0.0001)$ 


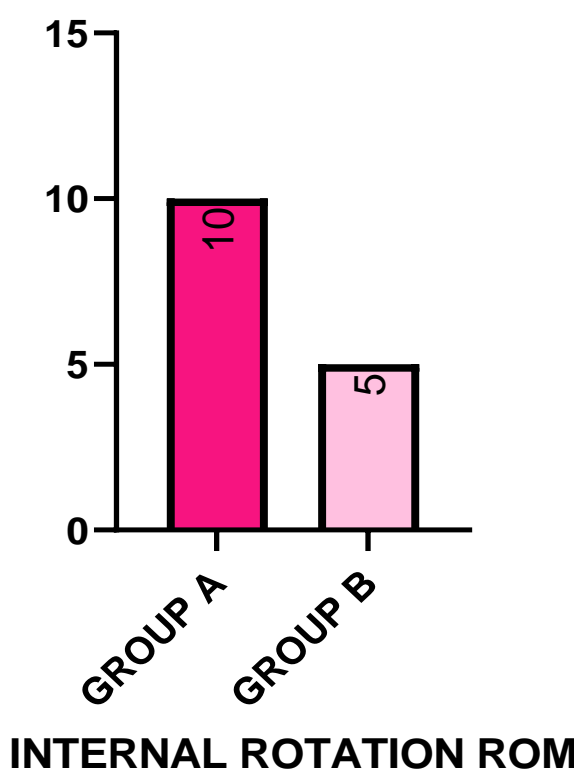

GROUP A (MULLIGAN MWM)

Graph 12- comparison of internal rotation ROM following Mulligan MWM and Kinesiotaping technique.

The above graph shows that there is significant increase in internal rotation following the application of MWM technique. $(\mathrm{p}<0.0001)$

\section{DISCUSSION}

30 subjects having Frozen Shoulder with limited range of motion in capsular pattern of age group 40 to 60 years were selected. They were randomly allocated into 2 groups i.e Group A and Group B consisting of 15 subjects each. Through this study we have tried to find the immediate effect of mulligan mobilization with movement compared to Kinesiotaping in frozen shoulder. Here, we documented changes in VAS and shoulder range of motion in our patients post the treatment.

Several treatments have been advocated for frozen shoulder. Mulligan mobilization with movement has been proved to be effective in getting an impaired joint back to its function by reversal of positional faults. This was supported by B. Chakradhar Reddy (2015) which concluded that mulligan's MWM is effective in the treatment of adhesive capsulitis. ${ }^{[1]}$

Graph 1 shows there is significant reduction in pre (median-5) and post (median -4) pain scores on VAS after the application of Mulligan's Mobilization with Movement technique. This finding is supported by Rizwan Haider et al (2014), where mulligan's Mobilization with movement technique significantly decreased pain scores in frozen shoulder patients and suggested that there was reduction in pain scores due to neurophysiologic effects on stimulation of peripheral mechanoreceptors and inhibition of nociceptors. The activation of apical spinal neurons as a result of peripheral mechanoreceptor by the joint mobilization produces presynaptic inhibition of nociceptive afferent activity. ${ }^{[10]}$ Graph 2, graph 3, graph 4 shows there is significant increase in shoulder abduction pre (median-100) and post (median-115), external rotation pre(mean32.133)and post(mean-46.00) and internal rotation pre (mean-46.80) and post(mean57.53) range of movement after the application of Mulligan's Mobilization with Movement technique. This finding is supported by Ujwal L Yeole (2017), where Mulligan's Mobilization with Movement technique significantly increased shoulder range of motion. The improvement in mulligan group, can be attributed to the corrective glide to achieve optimal alignment of the articular surfaces and its 
maintenance by appropriate recruitment of muscles by patient's active efforts. This goes well with the mulligan concept of positional fault. The alteration of shoulder biomechanics can be due to capsular tightness seen in adhesive capsulitis. This capsular tightness pulls the head of the humerus towards the glenoid fossa, thus altering humeral head in excursion in the glenoid. This glenohumeral mechanism alteration leads to altered mechanics of scapulothoracic and acromioclavicular joint which in turn leads to positional faults in these joints also. Mobilization have definite effect on this altered biomechanics. ${ }^{[8]}$

Graph 5 shows there is significant reduction in pre (mean- 5.8667) and post (mean-4.933) pain scores on VAS after the application of Kinesiotaping technique. This finding was supported by Smita Bhimrao Kanase (2012), where Kinesiotaping technique significantly decreased pain scores in frozen shoulder patients. Kinesiotape has expanding and contracting properties which provides gentle sensory stimulation to various types of sensory receptors in the skin during movement. This activates the spinal inhibitory system through stimulation of touch receptors and activates the descending inhibitory system to decrease pain via the Gate control theory. ${ }^{[4]}$

Graph 6, graph 7, graph 8 shows there is significant increase in shoulder abduction pre (mean-90.06) and post(mean98.133), external rotation pre(median-27) and post(median-35), internal rotation pre(mean-40.0) and post (mean- 46.4)range of movement after the application of Kinesiotaping technique. This finding was supported by Jung Hyun Choi et al(2017), where Kinesiotaping technique was effective in improving joint range of motion in patients with adhesive capsulitis. Kinesiotaping applied around the shoulder joint may have induced muscle contractions and stimulated the cerebellum as well as the reticular formation of the brainstem. As a result, the balance of muscle tension and bodily adjustment would be influenced reforming the joint range of motion. Kinesiotaping helps in restoring correct muscle function by supporting weakened muscles. ${ }^{[11]}$

According to the interpretation of graph 9, both the groups when compared, there was significant difference observed in both the groups with Group A (Mulligan's Mobilization with Movement) showing better reduction in pain when compared to Group B (Kinesiotaping). This was supported by Serdar Demirci et al (2017) where comparison of short term effects of mobilization with movement and Kinesiotaping on pain, function and balance in patellofemoral pain was done. In this study it was concluded that MWM showed better results as MWM technique is applied in the pain free movement along with active participation of the patient and therefore this change in mechanoreceptor perception leads to reorganisation and diminishes the patient's fear of movement. [12] Neurophysiologically, changes in central and descending pain processing mechanisms are probably involved. In addition large range of motion used in the application of MWM might alter concentration of inflammatory mediators and result in deactivation of nociceptors activated by such inflammatory mediators, while the pain relief and improvement of disability level is associated with MWM. ${ }^{[13]}$

According to the interpretation of graph 10, 11 and 12 when compared, there was significant difference observed with both the groups with Group A (Mulligan's Mobilization with Movement) shows greater increase in shoulder abduction, external rotation and internal rotation than Group B (Kinesiotaping). This was supported by Dr Gopal Nambi et al.(2012) where a comparative study was done between Kinesiotaping and Mulligan's mobilization with movement in subacute lateral ankle sprain in secondary school hockey players and it was concluded that an initial ameliorative effect was observed of mulligan's mobilization with movement technique on posterior talar glide and 
dorsiflexion range of motion in recurrent lateral ankle sprain. ${ }^{[14]}$ In MWM, the improvements could be because of biomechanical and neurophysiological mechanisms of MWM. Biomechanically, it was proposed that MWM may address joint partner bone alignment i.e. the alteration in shoulder biomechanics pulls the head of humerus towards the glenoid fossa thus altering the glenohumeral mechanism which leads to positional faults, which can be corrected through mulligan's mobilization with movement. In MWM patient receives simultaneous feedback of painless joint movements resulting in increased activity level. MWM helps in stretching and relaxing the capsule and muscles around the shoulder joint which might have resulted in improved motor performance, which would position the patient well to gain long term benefits. ${ }^{[13],[8]}$

On the basis of statistical reference, the present study supports the previous studies that both Mulligan Mobilization with Movement and Kinesiotaping show a significant increase in shoulder range of motion as well as reduction in pain on frozen shoulder.

And as proposed by Mulligan who states the effect of MWM's can be maintained further with self MWM which further enhance the lasting effect, and the inter group analysis helps us to conclude that Mulligan Mobilization with Movement shows better improvement in pain and range of motion than Kinesiotaping and hence Mulligan's MWM is a better technique of treatment than Kinesiotaping in the management of Frozen shoulder and hence it can be applied in clinical practice.

\section{CONCLUSION}

The present study concluded that Mulligan Mobilization With Movement is a better intervention on Frozen shoulder as it shows greater increase in shoulder range of motion and significant pain reduction when compared to Kinesiotaping technique.

\section{Clinical Implication}

Mulligan mobilization with movement can be used as an adjunct to an ongoing exercise program.

Mulligan mobilization with movement can be used to improve shoulder range of motion and alleviate pain.

\section{Abbreviations \\ MWM: Mobilization with movement \\ KT: Kinesiotaping \\ ROM: Range of motion \\ VAS: Visual Analogue scale}

\section{ACKNOWLEDGEMENTS}

I express my deep sense of gratitude and sincere thanks to our respected sir Dr. Ajay Kumar and Guide Dr. Leena Zore who immensely helped me with sincere guidance, untiring cooperation, valuable advice and endless inspiration during course of the study. I also take this opportunity to thank teaching and non-teaching staff, my friends and colleagues for their support.

\section{Conflict of Interest: None}

\section{Source of Funding: None}

\section{Ethical Approval: Approved}

\section{REFERENCES}

1. B. Chakradhar Reddy, Santosh Metgud. Mulligan's MWM and conventional therapy in adhesive capsulitis. Indian Journal of Physical Therapy. January-June 2015 volume-3 (Issue-1)

2. Pradeepshankar, Renukadevi .M, Nirnay Gowda, B.Punith, Harish Pai. Efficacy of Kinesiotaping as an adjunct to positional stretching of coracohumeral ligaments in patients with primary adhesive capsulitis. Innovative Journal of Medical and Health Science 3:2 March - April(2013) 45-51.

3. Hafiz Sheraz Arshad, Imtiaz Hussain Shah, Rashid Hafeez Nasir. Comparison of Mulligan Mobilization with Movement and End range Mobilization Following Maitland Techniques in patients with Frozen Shoulder . International Journal of Science and Research. April 2015 ; Volume 4 (Issue 4). 
4. Smita Bhimrao Kanase, S. Shanmugam. Effect of Kinesiotaping with Maitland Mobilization and Maitland Mobilization in management of Frozen Shoulder. International Journal of Science and Research . September 2014; Volume 3 Issue 9.

5. Gokhan Doner, Zeynep Guven, Ayçe Atalay, and Reyhan Celiker. Evaluation of Mulligan's Technique for Adhesive Capsulitis of The Shoulder. J Rehabil Med 2013;45:87-91.

6. Hemali j.Gandhi,Bhavna Mhatre, Leena Chilgar, Amrita Mehta. Effect of Taping on Scapular Posture and Shoulder Range of Motion In Subacromial Impingement Syndrome. International Journal Of Physiotherapy and Research . April 2019; Vol 7(2):3003-10.

7. Rahee Mulmulay and Himanshu Pathak. Effect of kinesiotaping Along with Kalternborn Mobilization in patients with subacute Adhesive Capsulitis . International Journal Of Current Advanced Research. December 2017; Volume 6; Issue 12; Page no. 8489-8498.

8. Ujwal L Yeole, Pratiksha D Dighe, Gaurai M Gharote, Rasika S Panse, Shweta Kulkarni \& Pournima A Pawar. Effectiveness of Movement with Mobilization In Adhesive Capsulitis of Shoulder: Randomized Controlled Trial. Indian Journal of Medical Research and Pharmaceutical Sciences; February 2017;4(2)

9. Ankit Shrivastava, Ashok K Shyam, Shaila Sabnis, Parag Sancheti. Randomised Controlled Study of Mulligan's versus Maitland's Mobilization Technique in Adhesive Capsulitis of Shoulder Joint. Indian Journal of Physiotherapy and Occupational Therapy; Oct.-Dec. 2011, Vol.5, No.4
10. Rizwan Haider, Ashfaq Ahmad, Saum-reZahra, Muhammad Kamran Hanif. To Compare Effects of Maitland and Mulligan's Mobilization Techniques in the treatment of Frozen Shoulder. Annals; JUL. - SEP.2014 VOL 20, ISSUE 3.

11. Jung Hyun Choi, Soon Hee Kim, Ho Jung An, Ja Pung Koo: Effects of 6 week Kinesiotaping and extracorporeal shock wave therapy on joint range of motion in patients with Frozen shoulder; ISSN 20928475, J Int Acad Phys Ther Res2017; 8(1):1095-1099.

12. Serder Demirci, Gizem Irem Kinikli, Michael Callaghan, Volga Bayrakci Tunay: Comparison of short term effects of mobilization with movement and Kinesiotaping on pain, function and balance in patellofemoral pain; Acta Orthopedica Et Traumatologica Turcica 51(2017)442-447

13. Priyesh P. Malgaonkar, Sai Kumar, Vinod Babu, Syed Rais Rizvi: short term effects of Mulligan's Mobilization versus Kinesiotaping on Knee pain and disability for osteoarthritis of knee; Int $\mathbf{J}$ Physiotherapy, Vol 1(4),233-240, October (2014); ISSN:2348-8336.

14. Dr Gopal Nambi S, Bijal Tarun Shah. Kinesiotaping versus Mulligan's mobilization with movement in subacute lateral ankle sprain in secondary school hockey players- comparative study. International Journal of Pharmaceutical Science and Health Care. April 2012; Volume 2, Issue 2.

How to cite this article: Shah KA, Zore L, Kumar A. Effect of mulligan mobilization with movement versus kinesiotaping in frozen shoulder. Int J Health Sci Res. 2021; 11(11): 37-47. DOI: https://doi.org/10.52403/ijhsr. 20211105 